\title{
Roles of Endogenous Angiotensin II or Kinin in the Mechanism of Altered Renal Vascular Responsiveness to Angiotensin II Following Acute Blood Volume Expansion in the Dog
}

\author{
Satoshi AKABANE, Shunichi KOJIMA*, Keiichi ITO* \\ and Minoru KAWAMURA \\ Department of Cardiovascular Dynamics, Research Institute and \\ * Department of Internal Medicine. National Cardiovascular Center. \\ Suita. Osaka 565. Japan \\ Accepted July 6, 1984
}

\begin{abstract}
To examine the role of endogenous angiotensin II or kinins in the mechanism of the increased renal vascular reactivity to exogenous angiotensin II following acute blood volume expansion, we examined whether captopril, a converting enzyme inhibitor, can prevent the increase in renal vascular response in anesthetized dogs. Pretreatment of dogs with captopril increased plasma renin activity, but it did not affect systemic blood pressure, renal blood flow and renal vascular resistance. Acute blood volume expansion with saline suppressed plasma renin activity in dogs with or without pretreatment with captopril. Basal level of renal vascular reactivity to angiotensin II was increased by pretreatment with captopril. In the control animals, acute blood volume expansion enhanced renal vascular reactivity to angiotensin II but not norepinephrine. The enhanced renal vascular reactivity to angiotensin 11, however, was not prevented by the captopril treatment. The failure of captopril to prevent an increase in renal vascular reactivity to angiotensin II following acute blood volume expansion was associated with an increase in urinary excretion of bradykinin. These data suggest that endogenous angiotensin $1 \mathrm{l}$ level is not necessarily a determinant for vascular reactivity to exogenous angiotensin 11 , especially in the case of acute blood volume expansion.
\end{abstract}

It is well-known that the vascular reactivity to exogenous angiotensin $\|$ is inversely related to plasma renin activity (1). Oliver and Cannon (2) reported that in the dog. renal vascular reactivity to angiotensin II was also changed when the endogenous activity of the renin angiotensin system was altered by varying sodium balance. They demonstrated that changes in renal blood flow (RBF) after intrarenal injections of subpressor doses of angiotensin II were inversely related to plasma renin activity. In addition, they showed that administration of a converting enzyme inhibitor (SQ 20.881) markedly enhanced renal vascular reactivity to angiotensin 11 in dogs with elevated endogenous angiotensin II levels by acute or chronic sodium depletion. whereas the same drug did not affect the renal vascular reactivity to angiotensin 11 in the dogs with suppressed plasma renin activity. These observations are compatible with the hypothesis that vascular reactivity to angiotensin $\mathrm{II}$ is determined by the numbers of receptor sites unoccupied by endogenous angiotensin 11 , as already proposed by Thurston and Laragh (3).

If this hypothesis is correct, the increase in vascular reactivity to angiotensin II after acute blood volume expansion with saline should also be blunted by the pretreatment of animals with a converting enzyme inhibitor. Unfortunately, however, such information is lacking in the paper of Oliver and Cannon (2) in spite of their comprehensive experimental protocols with other respects.

Therefore, the present study was designed 
to examine whether pretreatment of dogs with a converting enzyme inhibitor (Captopril) can prevent the enhancement of renal vascular reactivity to angiotensin $\|$ after acute saline loading in the dog. The results indicate that although the base-line responsiveness to angiotensin II was enhanced by the pretreatment with captopril, the enhancement of the reactivity by saline loading could not be prevented by the drug. suggesting that factors other than endogenous angiotensin $\|$ level are responsible for the mechanisms of altered renal vascular reactivity after acute saline loading.

\section{Materials and Methods}

Animal preparation: Either male or female mongrel dogs weighing from 10 to $21 \mathrm{~kg}$ were anesthetized with sodium pentobarbital $(30 \mathrm{mg} / \mathrm{kg}$, i.v.). After endotracheal intubation, respiration of dogs was assisted with a respirator. The left renal artery was exposed via the retroperitoneal route and denervated by severing and stripping the renal nerve, followed by application of $95 \%$ ethyl alcohol to the renal pedicle. Renal blood flow was measured by an electromagnetic flowmeter (NARCO RT-500). The femoral artery and cephalic vein were cannulated for measurement of systemic blood pressure and for i.v. administration of drugs and saline (154 mmoles// NaCl), respectively. Systemic blood pressure was measured with a Statham P23 Db pressure transducer and recorded with a Hewlett-Packard 7758 polygraph.
The left ureter was cannulated for collection of urine.

For infusion of angiotensin 11 or norepinephrine, the left renal artery was punctured at the site proximal to the flow probe with a fish-hook shaped 23 gauge needle connected to a polyethylene tube (PE-50). Renal vascular reactivity was assessed from the changes in renal blood flow after continuous intrarenal infusion of angiotensin $1 /$ (3 ng/ $\mathrm{kg} / \mathrm{min}$ ) or norepinephrine $(70 \mathrm{ng} / \mathrm{kg} / \mathrm{min})$. These agents were dissolved in saline and infused with an infusion pump at a rate of $0.35 \mathrm{ml} / \mathrm{min}$. Infusion of saline at this rate into the renal artery did not influence renal blood flow, and each dose of agents did not change the systemic blood pressure.

Experimental protocols (Fig. 1): The experiments were divided into two groups depending on the presence or absence of pretreatment with a converting enzyme inhibitor (CEI). They were labeled as the control and the CEl group, respectively. In the CEl group, a bolus i.v. injection of $1 \mathrm{mg} /$ $\mathrm{kg}$ captopril (SO 14.225) was followed by continuous i.v. infusion of the drug at 0.5 $\mathrm{mg} / \mathrm{kg} / \mathrm{hr}$ throughout the experiment. In preliminary studies, we confirmed that this dose of CEl was sufficient to suppress converting enzyme in vivo since either an increase in systemic blood pressure or a decrease in renal blood flow after bolus injection of angiotensin I was completely abolished by this dose of CEI. The following experimental protocol was similarly con-

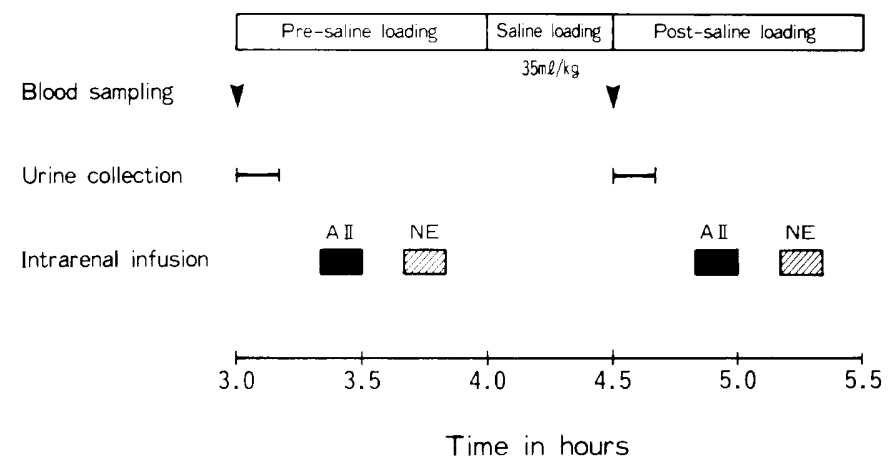

Fig. 1. Experimental protocol examining effect of acute blood volume expansion on renal vascular reactivity to angiotensin $\|$ and norepinephrine in control and CEI pretreated dogs. Angiotensin II and norepinephrine, were infused at rates of $3 \mathrm{ng} / \mathrm{kg} / \mathrm{min}$, and $70 \mathrm{ng} / \mathrm{kg} / \mathrm{min}$, respectively. 
ducted in both groups.

At about $3 \mathrm{hr}$ after the animal preparation was completed, the protocol was started as shown in Fig. 1. The protocol consists of 2 periods, pre- and post-acute saline loading. Under ordinary conditions, the saline was infused at a rate of $0.25 \mathrm{ml} / \mathrm{kg} / \mathrm{min}$, while during acute volume expansion it was increased at $35 \mathrm{ml} / \mathrm{kg} / \mathrm{min}$ for over $30 \mathrm{~min}$. At the initial time of each period, a $5 \mathrm{ml}$ aliquot of arterial blood was obtained from the brachial artery for measurements of plasma renin activity (PRA) and plasma aldosterone levels. It was followed by 10 min urine collection for measurements of urinary excretion of kallikrein and kinin. Then, renal vascular responses to infusion of angiotensin II and of norepinephrine were assessed successively at doses of $3 \mathrm{ng} / \mathrm{kg} / \mathrm{min}$ and $70 \mathrm{ng} / \mathrm{kg} / \mathrm{min}$, respectively.

Measurements of hormones and autacoids: Plasma renin activity (PRA) was determined by radioimmunoassay using a commercial kit (CEA-IRE-SOLIN Italy) based on the method of Haber et al. (4). Samples were incubated at $37^{\circ} \mathrm{C}$ for 1 or $2 \mathrm{hr}$, and PRA was expressed as $\mathrm{ng}$ of angiotensin $\mathrm{l} / \mathrm{ml} / \mathrm{hr}$. Plasma aldosterone concentration (PAC) was determined by radioimmunoassay using a commercial kit (CEA-IRE-SOLIN Italy) and was expressed as $\mathrm{pg} / \mathrm{ml}$.

Urinary kallikrein activity was assayed according to the method of Hitomi et al. (5). The activity was expressed as $10^{-3}$ esterase unit $(\mathrm{mEU}) / \mathrm{ml}$. Kinin concentration was measured according to the method of Ueno et al. (6) by an enzyme-immunoassay kit (MARKIT BK) which was obtained from Dainippon Pharmaceutical. To each $500 \mu l$ of urine sample was added 100 « 20\% TCA, and the protein precipitate was removed by centrifugation. The supernatant $(250 \mu)$ was neutralized by adding $250 \mu l$ of sodium phosphate- $\mathrm{NaOH}(\mathrm{pH} 8.0)$, and then $100 \mu l$ of the solution of anti-bradykinin antibody and $200 \mu$ of the solution of bradykinin labelled by $\beta$-D-galactosidase were added to $200 \mu l$ of this supernatant. The mixture was incubated at $0{ }^{\circ} \mathrm{C}$ for $30 \mathrm{~min}$. Then after addition of $200 \mu$ of $0.04 \mathrm{M}$ phosphate buffer ( $\mathrm{pH}$ 7.0) containing protein $A$, the mixture was further incubated at $0^{\circ} \mathrm{C}$ for
$30 \mathrm{~min}$. After centrifugation, the supernatant was incubated with $100 \mu$ of the substrate solution (25 mM 2-nitrophenyl- $\beta$-Dgalactopyranoside) at $30^{\circ} \mathrm{C}$ for $30 \mathrm{~min}$. The reaction was stopped with $2.5 \mathrm{ml}$ of $0.2 \mathrm{M}$ sodium phosphate- $\mathrm{NaOH}$ buffer ( $\mathrm{pH}$ 10.2), and the liberated 2 -nitrophenol was measured by it's absorbance at $410 \mathrm{~nm}$ with a spectrophotometer. The kinin concentration was expressed as $\mathrm{ng} / \mathrm{ml}$.

Statistical analysis: The data were expressed as means \pm S.E. Statistical analysis was performed by Student's $t$ test, either for paired or non-paired samples. $P$ values less than 0.05 were regarded as significant.

\section{Results}

Systemic blood pressure (SBP), renal blood flow (RBF), and renal vascular resistance $(R \vee R)$ were measured before and after blood volume expansion in both control and CEI groups. As summarized in Fig. 2, these parameters were unchanged after volume expansion in both groups. On the other hand, significant decreases in plasma renin activity (PRA) were observed after volume expansion in both groups, indicating that the degree of volume expansion was sufficient to suppress PRA. Pretreatment of animals with CEI markedly increased the basal PRA level, suggesting that this treatment

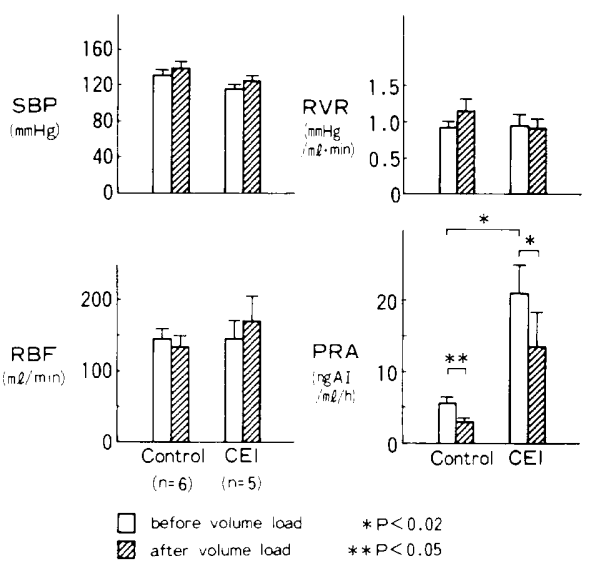

Fig. 2. Influence of saline infusion $(35 \mathrm{ml} / \mathrm{kg}$ ) on systemic and renal hemodynamics and PRA. Vertical lines indicate mean \pm S.E. SBP, systemic blood pressure; RBF, renal blood flow: RVR renal vascular resistance: PRA, plasma renin activity. 
was sufficient to shut off the negative feedback loop of renin release via angiotensin II. Figure 3 summarizes the data of experiments in which renal vascular responses to angiotensin $\|$ and norepinephrine were examined before and after blood volume expansion in control and CEI groups. In the control group, blood volume expansion increased renal vascular reactivity to angiotensin II without affecting the reactivity to norepinephrine. In the CEI group, renal vascular reactivity to angiotensin II was increased before volume expansion as compared to the basal response in the control group. However, the reactivity to angiotensin Il was further increased after blood volume was expanded by saline infusion. The change in renal vascular reactivity was selective for angiotensin $\mid \mathrm{l}$ because the reactivity to norepinephrine was unaltered. These observations indicate that blood volume expansion increases renal vascular reactivity to exogenous angiotensin 11 even under the condition in which conversion of endogenous angiotensin I to angiotensin $\mathrm{II}$ is completely blocked.

In order to examine whether changes in circulating aldosterone level or the kallikreinkinin system are responsible for this altered renal vascular responsiveness to angiotensin Il after blood volume expansion. we measured

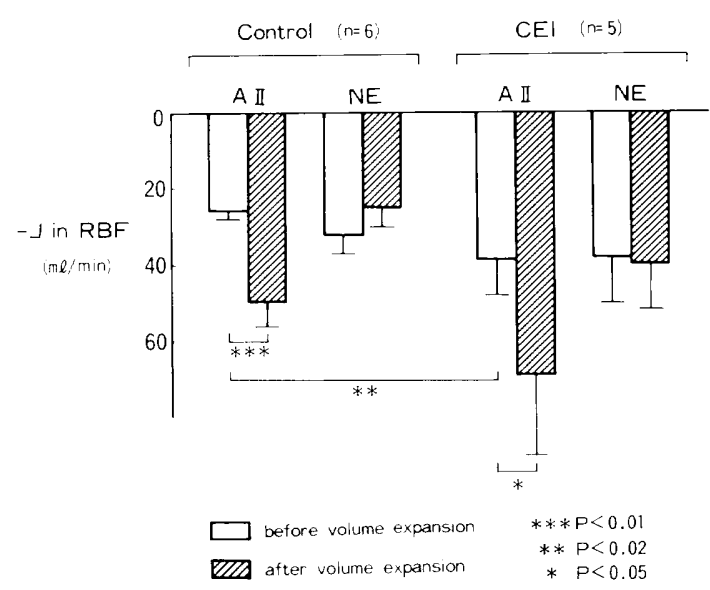

Fig. 3. Effects of volume expansion (saline infusion of $35 \mathrm{ml} / \mathrm{kg}$ ) on the renal vascular response to angiotensin || and riorepinephrine (NE). Vertical lines indicate the mean \pm S.E. plasma aldosterone level, urinary excretion of kallikrein and bradykinin simultaneously. The data are summarized in Fig. 4. Plasma aldosterone levels were significantly reduced after blood volume expansion in both the control and the experimental group. The basal plasma aldosterone level of the CEI group was not different from that of the control group. This is seemingly discrepant with the fact that endogenous angiotensin II was suppressed by pretreatment with CEI. The reason for this discrepancy is unknown at the present time. Delayed response of aldosterone synthesis may not account for this discrepancy, since acute blood volume expansion reduced plasma aldosterone level during the experimental period.

Urinary excretion of kallikrein was unaffected by volume expansion in both groups

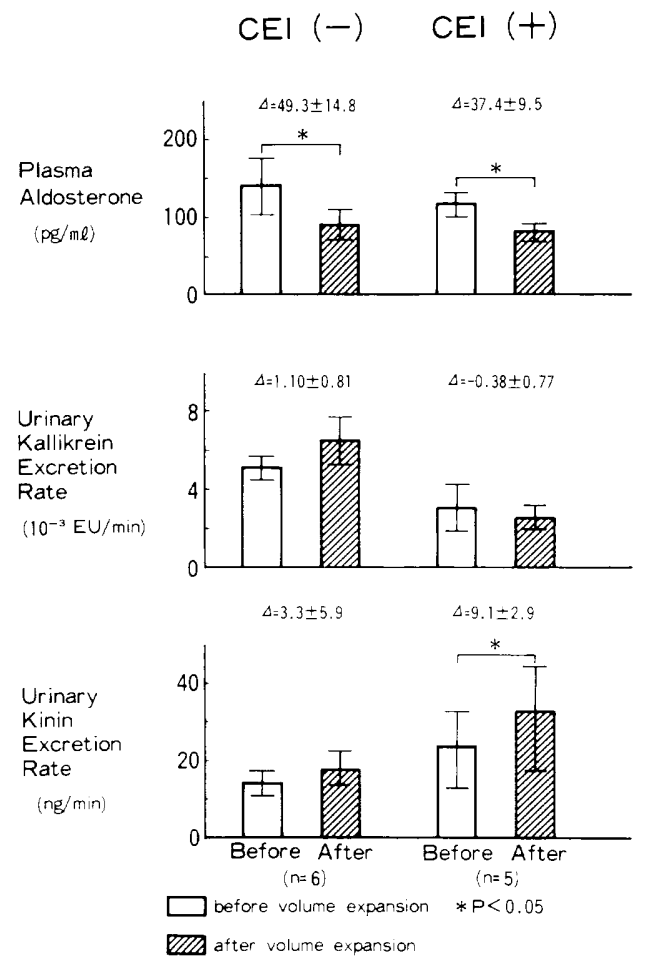

Fig. 4. Effects of volume expansion (saline infusion of $35 \mathrm{ml} / \mathrm{kg}$ ) and angiotensin-coverting enzyme inhibition by captopril on plasma aldosterone concentration and urinary kallikrein and kinin excretion. Vertical lines indicate the mean \pm S.E. $I$ means difference of the values before and after blood volume expansion in paired samples. 
(Fig. 4). Urinary excretion rate of kallikrein tended to be lower in the CEI group, but statistically, this was not significant. In the control group urinary excretion of bradykinin was not affected by blood volume expansion. whereas in the CEI group, it was increased significantly. The basal level of the urinary excretion rate of bradykinin tended to increase by the pretreatment with CEl, but this increase was not significant because of a large scatter of the data in the CEI group.

\section{Discussion}

The present study confirmed the previous observations by Oliver and Cannon (2) that acute blood volume expansion enhanced renal vascular response to angiotensin 11 . This effect was specific for angiotensin II. since the response to norepinephrine was unchanged after acute volume expansion. The purpose of the present study was to examine roles of endogenous angiotensin 11 levels or kinin in the mechanism of altered renal vascular responsiveness to angiotensin Il following acute blood volume expansion. For this purpose, we used captopril, a converting enzyme and kininase $\|$ inhibitor.

A comprehensive study on this issue has already been reported by Oliver and Cannon (2). who also examined the effect of CEI on renal vascular response to angiotensin II under conditions of varying sodium balance. including acute and chronic sodium depletion. and chronic sodium excess. They showed that treatment with $\mathrm{CEI}$ enhanced renal vascular response to angiotensin II in acute and chronic sodium depletion in which plasma renin activity was elevated, whereas it did not change the renal vascular response to angiotensin 11 in chronic sodium excess in which plasma renin activity was suppressed. These observations are in accord with the hypothesis that endogenous angiotensin 11 levels are the determinant of the vascular responsiveness to angiotensin II.

If the same mechanism can hold for the enhanced vascular response after acute volume expansion, pretreatment of animals with CEI should prevent alteration of the vascular responsiveness after acute blood volume expansion. The present study clearly shows that this is not the case. Renal vascular response to angiotensin II was increased after volume expansion in dogs pretreated with CEI. Since it is reasonable to assume that the circulating level of angiotensin II is diminished in CEI treated dogs, the increased vascular reactivity after volume expansion may not be accounted for by the changes in circulating angiotensin II.

It should be noted that urinary excretion rate of bradykinin was increased after acute blood volume expansion in the CEI group. but not in the control group. Although it is unknown why urinary excretion of bradykinin is increased after volume excretion only in the CEI group, it is possible that an excess of bradykinin, a vasodilator system, may enhance responsiveness to angiotensin II, thereby cancelling the expected diminution of renal vascular responsiveness to angiotensin II in the CEI group. This possibility has been carefully excluded by Oliver and Cannon (2) who showed that renal vascular response to angiotensin $\|$ as well as to norepinephrine was unaffected during bradykinin infusion. However, it is still possible that endogenous, but not exogenous, bradykinin plays a role in the increased renal vascular responsiveness to angiotensin II following acute blood volume expansion. Exogenous bradykinin is never excreted in urine (7). Urinary bradykinin originates exclusively from the kidney. Thus it is reasonable to assume that sites of action of endogenous renal bradykinin may differ from those of exogenous bradykinin.

Recently, Jandhyala and Hom (8) reported that in nephrectomized dogs, acute blood volume expansion enhanced responsiveness of vascular smooth muscle of perfused hind limb preparation to norepinephrine, but not to angiotensin 11 . This is seemingly contradictory to our observation. However, it is possible that renal vessels respond differently because of the local effect of the kallikreinkinin system.

In summary, we have demonstrated that the pretreatment of dogs with captopril did not prevent the enhanced renal vascular reactivity to angiotensin $\|$ following acute blood volume expansion, suggesting factors other than endogenous angiotensin II level are responsible for this phenomenon. An 
increase in endogenous renal bradykinin may be one of the candidates responsible for this phenomenon. However, possible contribution of other factors such as various kinds of natriuretic hormones can not be ruled out in this study.

Acknowledgments: We thank Etsuko Kakimi for skillful technical assistance. We indebted to Dr. Masashi Imai for complete revision of this manuscript. This work was supported in part by grants from the Ministry of Education. Science and Culture, Japan.

\section{References}

1 Kaplan, N.M. and Silah, J.G.: The effect of angiotensin II on the blood pressure in humans with hypertensive disease. J. Clin. Invest. 43, 659-669 (1964)

2 Oliver, J.A. and Cannon, P.J.: The effect of altered sodium balance upon renal vascular reactivity to angiotensin $\|$ and norepinephrine in the dog. J. Clin. Invest. 61, 610-623 (1978)

3 Thurston, H. and Laragh, J.H.: Prior receptor occupancy as a determinant of the pressor activity of infused angiotensin 11 in the rat. Circ. Res. 36, 113-117 (1975)

4 Haber, E., Koerner, T., Page, L.B., Kliman, B. and Purnode, A.: Application of a radioimmunoassay for angiotensin 1 to the physiologic measurements of plasma renin activity in normal human subjects. J. Clin. Endocrinol. Metab. 29, 1349-1355 (1969)

5 Hitomi, Y., Niinobe, M. and Fujii, S.: A sensitive colorimetric assay for human urinary kallikrein. Clin. Chim. Acta 100, 275-283 (1980)

6 Ueno, A., Oh-ishi, S., Kitagawa, T. and Katori, M.: Enzyme immunoassay of bradykinin using $\beta$-Dgalactosidase as a labeling enzyme. Biochem. Pharmacol. 30, 1659-1664 (1981)

7 Abe, K.: Urinary excretion of kinin in man with special reference to its origin. Tohoku J. Exp. Med. 87, 175-184 (1965)

8 Jandhyala, B.S. and Hom, G.J.: Effects of acute blood volume expansion on vascular resistance and reactivity in anesthetized dogs. Clin. Sci. 65, 9-17 (1983) 